\title{
Application of Statistical Analysis to DC-DC Converters
}

\author{
Oliver Woywode and Henry Güldner \\ Elektrotechnisches Institut \\ Technische Universität Dresden \\ D-01062 Dresden, Germany \\ woywode@ieee.org
}

\begin{abstract}
This paper aims at showing the benefits of a statistical analysis applied to DC-DC converters. A boost converter under current-mode control operating chaotically was being used as an example. Theoretical results are verified by measurements. Parallels to results other researchers are drawn. New simple expressions for the DC value of the boost's input current and the AC power are proposed. The statistical approach covers the periodic and aperiodic behavior quite naturally. It reveals that almost all $\mathrm{AC}$ power is contained in the chaotic part of the input current. Impacts on meeting electromagnetic interference regulations are suggested.
\end{abstract}

Key words: DC-DC Converter, Chaos, Statistical Analysis

\section{Introduction}

Recently, statistical analysis has attracted some attention in power electronics [3], [6], [9]. Randomized or chaotic modulation schemes are able to reduce the peaks of the switching harmonics. This is important since all electrical and electronic devices in the European Community have to meet certain electromagnetic interference (EMI) requirements. The specified EMI regulations actually favour a broadband spectra over a narrow one. The idea of operating DC-DC converter chaotically was proposed in order to ameliorate power supply interference since spectral spreading comes naturally with chaos [3], [4]. The advantage with respect to size and cost is evident.

In Section 2 the boost converter used throughout this paper and its embedded map is introduced very briefly. Section 3 analyzes the map statistically and highlights the straightforward determination of the Lyapunov exponent. Section 4 calculates the $D C$ value of the input current and the $\mathrm{AC}$ power based on the moments of the map and compares the values with experimental results. Section 5 analyzes the AC power associated with the input current ripple.

\section{Chaotic Boost Converter}

Fig. 1 shows the current programmed PWM boost converter used to verify the analytical results. The primary control variable is the reference current $I_{r}$. By changing this parameter while keeping the load resistance constant, i. e. changing the conversion voltage ratio, the converter may operate chaotically. Chaotic behavior is typical for DC-DC converters under currentmode control if operated with a duty cycle larger than

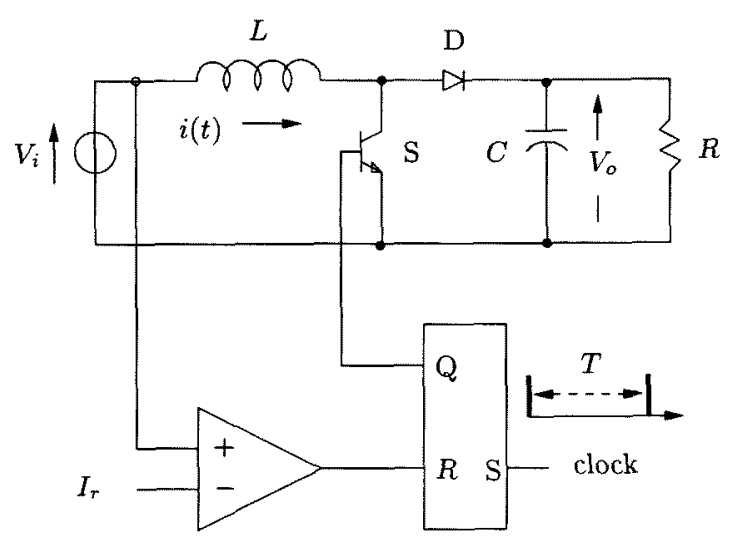

Figure 1. Boost converter. $[T]=100 \mu \mathrm{s},[L]=1 \mathrm{mH}$, $[C]=0.16 \mathrm{mF},[R]=92 \Omega,\left[V_{i}\right]=10 \mathrm{~V}$.

one half. Traditionally, this behavior has been regarded as unstable. It was consequently prevented by a stabilizing ramp in the inner current loop. This paper omits the ramp in the analysis and in the practical design.

Practically, the reference variable is adjusted by a voltage feedback loop. As the dynamics of this feedback is slow compared to the frequency of the switching process, $I_{r}$ will be assumed constant throughout this paper. The converter is peak current controlled. As long as the switch is closed the input (inductor) current $i(t)$ increases linearly until it reaches $I_{r}$. All clock impulses $T$ are ignored during this time. The switch opens and the current decreases until the next clock pulse causes the switch to close.

If the voltage feedback loop perfectly accomplishs its goal a constant output voltage $V_{o}$ may be assumed. 
Then the input or inductor current of the boost converter is given by

$$
i(t)= \begin{cases}I_{n}+c_{1}\left(t-\tau_{2 n}\right) & \tau_{2 n}<t<\tau_{2 n+1}, \\ I_{r}+c_{2}\left(t-\tau_{2 n+1}\right) & \tau_{2 n+1}<t<\tau_{2(n+1)}\end{cases}
$$

where the slopes $c_{1}$ and $c_{2}$ are defined by

$$
c_{1}=V_{i} / L \text { and } c_{2}=\left(V_{i}-V_{o}\right) / L
$$

for the on and off interval, respectively. The current waveform is depicted in Fig. 2 indicating continuousconduction mode.

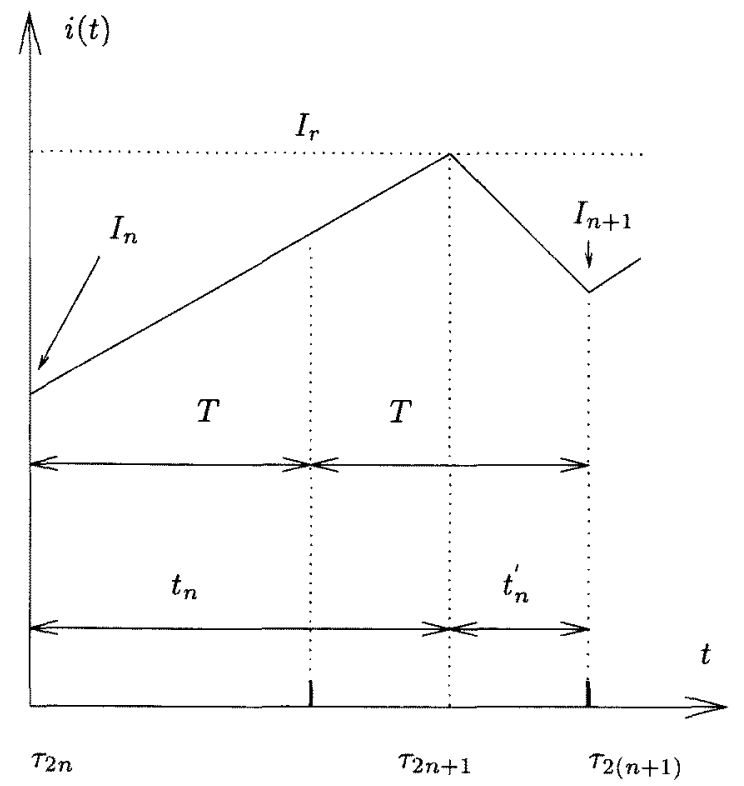

Figure 2. Chaotic waveform of boost's input current in continuous-conduction mode.

Using the relations illustrated in Fig. 2

$$
t_{n}=T x_{n}, \quad t_{n}^{\prime}=T\left(1-x_{n} \bmod 1\right)
$$

the one-dimensional discrete return map embedded in the dynamical system may be derived as

$$
x_{n+1}=g\left(x_{n}\right)=\alpha\left(1-x_{n} \bmod 1\right) .
$$

The modulo function returns the fractional part of a number. Thus, every number may be represented by

$$
x=x \bmod 1+\lfloor x\rfloor
$$

where the floor function $\lfloor\cdot\rfloor$ gives the greates integer less than or equal to a number.

The map $g$ defined by (4) is the Renyi Map. It approximates the dynamics of the converter sufficiently. This result has already been reported in [1], [2], [3]. The parameter $\alpha$ characterizes the voltage ratio of the converter

$$
\alpha=V_{o} / V_{i}-1 \text {. }
$$

Since $V_{o}>V_{i}$ holds for a boost, $\alpha$ is positive. For $\alpha$ larger than one the map (4) is chaotic. This corresponds to a duty cycle larger than one half.

\section{Statistical Analysis}

For this PWM boost converter under current-mode control, the statistical analysis boils down to the corresponding analysis of the embedded discrete return map (4). The statistical analysis models the input current as an impulse process $y(t)$ by taking its second derivative

$$
y(t)=\frac{\mathrm{d}^{2} i}{\mathrm{~d} t^{2}}=\frac{V_{o}}{L} \sum_{n=0}^{\infty}(-1)^{n} \delta\left(t-\tau_{n}\right) .
$$

This transforms the input current into a sequence of delta functions of area $V_{o} / L$. This area is the difference between the slopes $c_{1}$ and $c_{2}$. For $\alpha$ larger than one the sequence will be chaotic. The positive delta functions occur at even $\tau_{n}$, i.e. they coincide with the multiples of the clock impulses that cause the switch to close. These occurrences are generated by the map (4). The scenario is illustrated in Fig. 3.

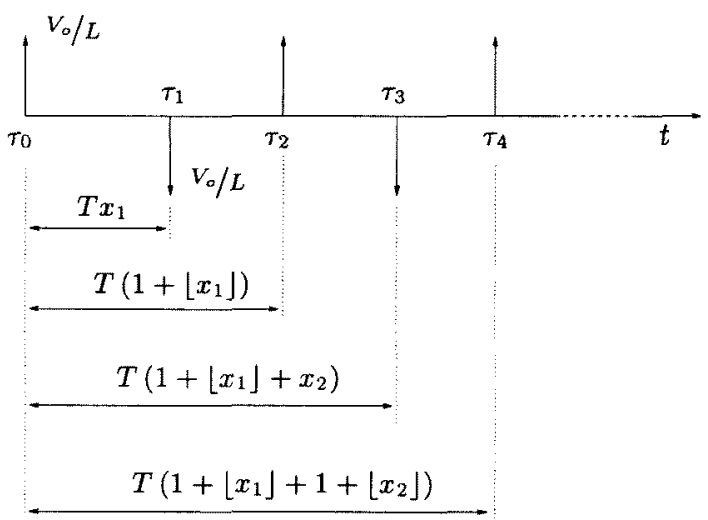

Figure 3. Chaotic impulse process.

The chaotic impulse process is ergodic. Therefore, time averages may be replaced by ensemble averages with respect to the invariant density of the Renyi Map that governs the chaotic impulse process. The Rényi Map possess an invariant density $p(x)$ that is absolutely continuous with respect to the Lebesgue measure on the interval $[0, \alpha)$. This density is ergodic, unique and asymptotically stable [3]. The evolution of this density is governed by the Frobenius-Perron-Operator (FPO), generally defined by

$$
p_{n+1}(x)=\int_{0}^{\alpha} \delta[x-g(\tilde{x})] p_{n}(x) \mathrm{d} \tilde{x} .
$$

The FPO of the Rényi Map is denoted by the symbol $\mathcal{P}$ and explicitely given by

$$
\mathcal{P} p(x)=\sum_{n=1}^{n(x)} \frac{1}{\alpha} p\left(n-\frac{x}{\alpha}\right)
$$


with

$$
n(x)= \begin{cases}\lfloor\alpha\rfloor & \text { if } \quad x \leq g(\alpha) \\ \lfloor\alpha\rfloor+1 & \text { if } \quad x>g(\alpha)\end{cases}
$$

The invariant density is the fixed point of the FPO and may be obtained by iterated action of the FPO on any initial density $p_{0}(x)$

$$
p(x)=\lim _{n \rightarrow \infty} \mathcal{P}^{n} p_{0}(x) .
$$

The initial density may be any nonnegative function with integral one. The invariant density is piecewise constant and determines the evolution of the inductor current samples via the map. All relevant properties of the current may be expressed with respect to the embedded map.

Recent findings about border-collision bifurcations in DC-DC converters suggest that the sudden onset of chaos is typical for these systems [10]. The statistical approach indicates the transition compactly through the Lyapunov exponent

$$
\forall \alpha: \quad \lambda_{g}=\mathcal{E}(\ln |\mathrm{d} g / \mathrm{d} x|)=\ln \alpha .
$$

The operation $\mathcal{E}(\cdot)$ stands for computing the mathematical expectation with respect to the invariant density. At a glance

$$
\forall \alpha>1: \quad \lambda_{g}>0,
$$

indicating chaos. This seems to be more accessible than solving a system of nonlinear equations in order to determine the critical value of the bifurcation parameter based on the A-switching map as suggested by [5].

\section{Moments of the Current}

In [1], the first moments of the map have been explicitly calculated for integer $\alpha$

$$
m_{k}(\alpha)=\mathcal{E}\left(x_{n}^{k}\right)=\frac{\alpha^{k}}{k+1}, \quad k=1,2 \ldots
$$

The DC value of the input current may be calculated from these moments

$$
I=\mathcal{E}[i(t)]=I_{r}-\frac{T V_{i}}{2 L} \frac{m_{2}(\alpha)}{m_{1}(\alpha)} .
$$

For integer $\alpha$ this expression simplifies to

$$
I=I_{r}-\alpha T V_{i} /(3 L) .
$$

Note that (16) is identical to a recently published result $^{1}[7],[8]$ repeated here for convenience

$$
I_{r}=(1+\alpha)^{2} \cdot V_{i} / R+\alpha T V_{i} /(3 L)
$$

\footnotetext{
${ }^{1} \mathrm{~A}$ charge balance approach was being used.
}

In order to highlight the identity, substitute (6) appropriately into $(1+\alpha)^{2} V_{i} / R$ yielding

$$
\left(V_{o} / V_{i}\right)^{2} \cdot V_{i} / R=P_{o} / V_{i} .
$$

In an ideal converter the output power $P_{o}$ is equal to the input power $P_{i}$. Therefore,

$$
P_{o} / V_{i}=P_{i} / V_{i}=I_{i}=I \text {. }
$$

Accordingly, the DC value of the input current must be

$$
I=(1+\alpha)^{2} \cdot V_{i} / R
$$

Back-substituting (20) in (17) shows the identity to (16).

In the periodic case $(\alpha<1)$ the concept of the duty cycle $d=t_{n} / T$ may be invoked. The duty cycle is a stable fixed point of the map (4). The input-output voltage ratio is well-known in that case

$$
V_{o} / V_{i}=1 /(1-d) \text {. }
$$

According to (6) the following relation between $d$ and $\alpha$ holds

$$
1 /(1-d)=1+\alpha
$$

Inserting (22) in (20) leads to the well-known equation for the DC value of the input current in the periodic case

$$
I=1 /(1-d)^{2} \cdot V_{i} / R
$$

Thus, (20) is identical to a result long known from statespace averaging in the periodic case. Note however, that the duty cycle concept may not be extended to aperiodic operation. This underlines the benefits of the statistical approach.

Fig. 4 compares (20) with measurements. The agreement is evidently satisfactory. The $\mathrm{DC}$ value of the input current has been directly measured. To apply (20), (17) is solved for $\alpha$ and a given $I_{r}$. The control variable $I_{r}$ is known from the experimental setup.

When comparing (16) and (20) one should bear in mind that the feedback control introduces a dependence between $\alpha$ and $I_{r}$. This issue was alluded to in [7].

Although (16) and (20) are exact only for integer $\alpha$ they reasonably estimate the periodic $(\alpha<1)$ and the aperiodic case $(\alpha>1)$. A detailed comparison between the integer and non-integer case for (16) was made in [1]. The estimate is good up to a certain $\alpha$ as parasitics (such as copper losses of the inductance) are not considered. This becomes also apparent in Fig. 4 The deviation grows as $\alpha$ increases.

Provided the output voltage has been measured, there is the direct way of calculating $\alpha$ from (6). If $\alpha$ is determined that way and then used in (16) the result is slightly larger than the corresponding value in Fig. 4. If the same $\alpha$ is inserted in (20) the result is slightly less than the one in Fig. 4. 


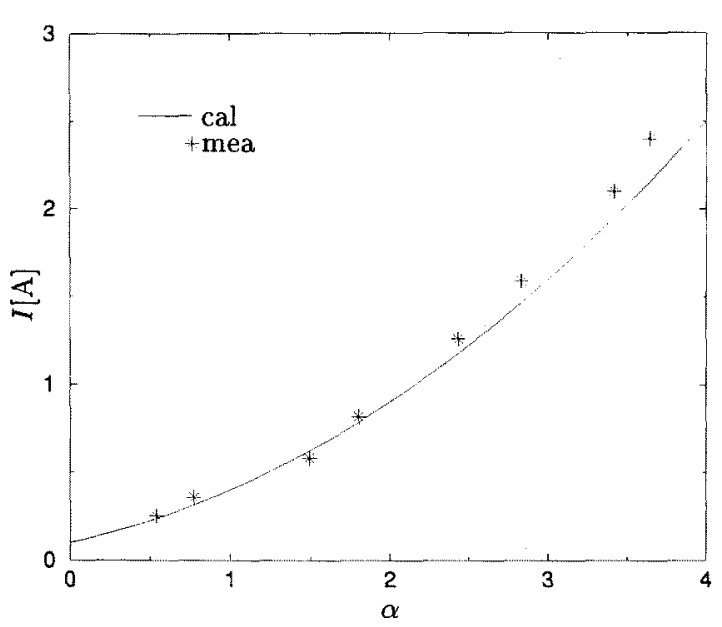

Figure 4. Calculated DC value of inductor current according to (20) and measurement.

The discrepancy may be relaxed by considering parasitic effects. For example, taking the copper losses of the incuctance $R_{L}$ into account, (6) becomes

$$
(1+\alpha) \sqrt{1-\frac{R_{L}}{R}(1+\alpha)^{2}}=\frac{V_{o}}{V_{i}} .
$$

The procedure mentioned above has been repeated with $R_{L}=0.19 \Omega$. The disagreement reduces further. This supports the conclusion that considering all parasitic effects gives a consistent picture.

In order to consider design issues, EMI regulations and hence filtering issues the $\mathrm{AC}$ power associated with the ripple of the inductor current is crucial. An analytical expression for the $\mathrm{AC}$ power that corresponds to the variance of the input current has been derived in [1]

$$
P_{A C}=\mathcal{E}\left\{[i(t)-I]^{2}\right\} \approx \frac{1}{18}\left(\frac{T V_{i}}{L} \alpha\right)^{2} .
$$

The approximation in (25) becomes exact for integer $\alpha$. Fig. 5 compares (25) and the measured AC power. The agreement is remarkable, suggesting that (25) is a sufficient approximation for all $\alpha$. The deviation apparently increases with growing $\alpha$. Again, this is due to the parasitic effects not considered in (25). If $\alpha$ stems from (6) the $\mathrm{AC}$ power will be slightly less than the one shown in Fig. 5.

The rms value of the current through the output capacitor has been measured. The squared rms value corresponds to the power delivered to a one $\mathrm{Ohm}$ resistance. This assumes that only $\mathrm{AC}$ current passes through the capacitor.

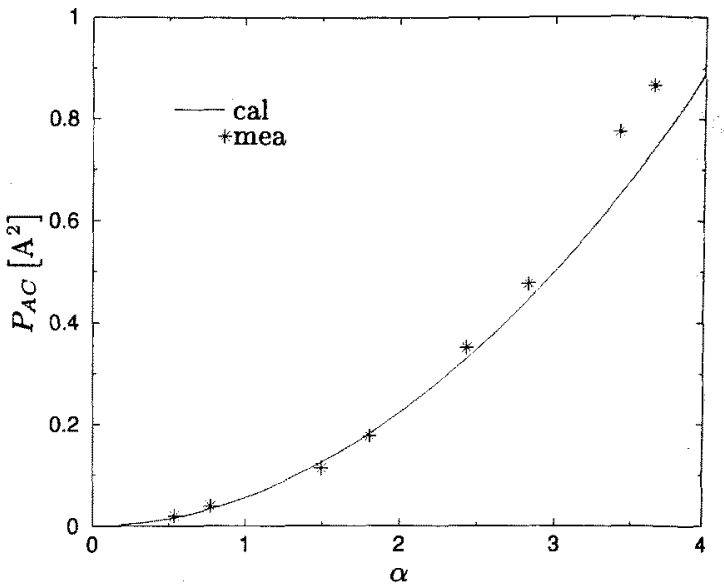

Figure 5. Calculated $\mathrm{AC}$ power associated with ripple of inductor current according to (25) and measurement.

\section{AC Power Analysis}

One of the major advantages of the statistical approach is its ability to analyze the AC power via the power density spectrum (PDS). The periodic part of the PDS, i.e. the PDS at the clock frequency and its harmonics, was calculated in [3]. Explicit analytical expressions for the PDS at all frequencies were presented in [1], facilitating the further investigation of the $\mathrm{AC}$ power. The way to arrive at the PDS is summarized in the following.

The PDS of the input current $S(\omega)$ may be calculated from the PDS of the chaotic impulse process $S_{\delta}(\omega)$ because they are related to each other by linear timeinvariant operations [9]

$$
S(\omega)=\frac{S_{\delta}(\omega)}{\omega^{4}}=\frac{1}{\omega^{4}} \cdot \lim _{T^{*} \rightarrow \infty} \frac{\mathcal{E}\left(\left|Y_{T^{*}}(\mathrm{j} \omega)\right|^{2}\right)}{T^{*}}
$$

where

$$
Y_{T^{*}}(\mathrm{j} \omega)=\int_{0}^{T^{*}} y(t) \mathrm{e}^{-\mathrm{j} \omega t} \mathrm{~d} t
$$

is the Fourier transform of the impulse process in Fig. 3. The upper limit $T^{*}$ denotes the expectation value of the total time $T_{N}$ for a large number $N$ of switching cycles

$$
T^{*}=\mathcal{E}\left[T_{N}\left(x_{n}\right)\right]=N \mathcal{E}\left(T_{s i}\right)
$$

Then

$$
\forall T^{*} \quad \exists N: \quad T_{N}\left(x_{n}\right)<T^{*}<T_{N+1}\left(x_{n}\right)
$$

and

$$
T_{N}\left(x_{n}\right)=T \sum_{n=1}^{N} 1+\left\lfloor x_{n}\right\rfloor
$$


Note the correspondence of (30) to Fig. 3. The expectation value of the $\mathrm{i}^{\text {th }}$ switching interval $\mathcal{E}\left(T_{s i}\right)$ may be calculated via the first moment of the Renyi Map, i e. an ensemble based expectation

$$
\begin{aligned}
\mathcal{E}\left(T_{s i}\right) & =T \int_{0}^{\alpha}(1+\lfloor x\rfloor) p(x) \mathrm{d} x \\
& =T(1+1 / \alpha) m_{1}(\alpha)=\lim _{N \rightarrow \infty} \frac{T_{N}\left(x_{n}\right)}{N} .
\end{aligned}
$$

After some manipulation the PDS of the impulse process may be written as

$$
\begin{aligned}
S_{\delta}(\omega)= & \frac{V_{0}^{2}}{L^{2}} \frac{2}{\mathcal{E}\left(T_{s i}\right)}\left\{1+2 \lim _{N \rightarrow \infty} \sum_{n=1}^{2 N-2}(-1)^{n}\right. \\
& \left.\cdot(1-(n+1) / 2 / N) \operatorname{Re}\left[\Theta_{r_{n}}(\omega)\right]\right\}
\end{aligned}
$$

where

$$
\Theta_{\tau_{n}}(\omega)=\mathcal{E}\left(e^{j \omega \tau_{n}}\right)
$$

are the characteristic functions, i.e. the Fourier transforms of the densities $p\left(\tau_{n}\right)$ of the switching instants $\tau_{n}$. Fig. 6 compares the numerical implementation of (26) and (32) with measurements for $\alpha=2.1$. The

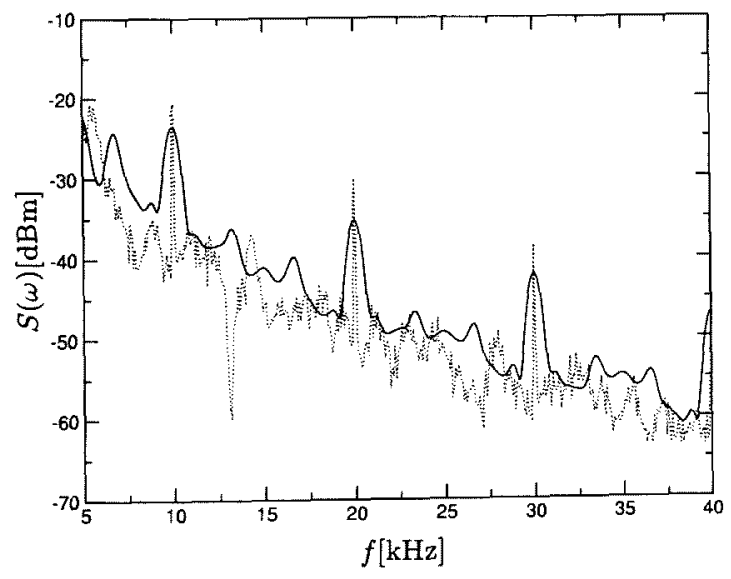

Figure 6. Calculated (straight line) and measured (dashed line) power density spectrum of inductor current for $\alpha=2.1$.

agreement is acceptable, although the numerical code does not predict the negative spike at about $13 \mathrm{kHz}$.

The PDS may be separated in a periodic (discrete) and an aperiodic (continuous) part. The periodic part of the AC power is calculated from the periodic part of the PDS of the input current

$$
P_{p}=\frac{1}{2 \pi} \int_{-\infty}^{\infty} S_{p}(\omega) \mathrm{d} \omega
$$

which for integer $\alpha$ is given by

$$
P_{p}=\frac{1+\alpha}{360 \pi}\left(\frac{T V_{i}}{L}\right)^{2}
$$

The ratio $P_{p} / P_{A C}$ converges rapidly toward zero. Almost all $A C$ power must be in the chaotic part of the input current at very low frequencies. This may impact on the filter design of DC-DC converters.

\section{Conclusions}

This paper applied a rigorous statistical approach to analyze a boost converter. Theoretical results based on this approach were validated by measurements and compared with results other researchers. It has been shown that statistical analysis may be a powerful tool in power electronics. The analysis emphasizes that chaotic operation does not need to be avoided. Simple analytical expressions for the Lyapunov exponent, the $\mathrm{DC}$ part of the input current and for the $\mathrm{AC}$ power are presented. The latter compare favourably with our measurements. The AC power can be split in a periodic and a clearly dominating aperiodic part.

The analysis of the inductor current PDS reveals that most of the power is being pushed to low frequencies if the converter operates chaotically. Due to the nature of the PWM control scheme the discrete part of the spectrum does not vanish completely if $\alpha$ gets larger. The power contained therein may be neglected for a practical design, however. This is certainly beneficial for meeting EMI regulations, since they are strict in the radio frequency band but much more relaxed at lower frequencies.

On the other hand, the power shift to lower frequencies causes the inductor current and capacitor voltage ripple to become larger. The power may even be concentrated at frequencies that fall in the passband of the boost converter's output filter, possibly inducing a not acceptable output voltage ripple. Clearly, a careful trade-off between time-domain (ripple) and frequency-domain (EMI) performance is required. The fundamental advantage of chaotic operation of dc$\mathrm{dc}$ converters over periodic operation is that such a trade-off is at all a viable option. This is because periodic operation aims at the best possible time-domain performance but is forced to accept the worst frequencydomain performance. The required compliance with EMI standards then calls for expensive filtering efforts.

\section{Acknowledgement}

The authors gratefully acknowledge Hajo Sgamlin's assistance with experimental setup and measurements.

(Manuscript received March 17, 2000, revised Jan. 9, 2001) 


\section{References}

[1] A. L. Baranovski, A. Mögel, W. Schwarz, and O. Woywode. Statistical analysis of a dc-dc converter. In Workshop on Nonlinear Dynamics of Electronic Systems, NDES 1999, pages 77-80, Rønne, Bornholm, Denmark, July 1999.

[2] J. H. B. Deane. From circuits to spectral peaks. In International Symposium on Circuits and Systems, ISCAS 1999, pages $\mathrm{V}-274-\mathrm{V}-277$, Orlando, Florida, USA, May/June 1999.

[3] J. H. B. Deane, P. Ashwin, D. C. Hamill, and D. J. Jefferies. Calculation of the periodic spectral components in a chaotic dc-dc converter. IEEE Trans. Circuits and Systems - I: Fundamental Theory and Applications, 46(11):1313-1319, November 1999.

[4] J. H. B. Deane and D. C. Hamill. Improvement of power supply emc by chaos. Electronics Letters, 32(12):1045, June 1996.

[5] M. di Bernardo, F. Garofalo, L. Glielmo, and F. Vasca. Switchings, bifurcations, and chaos in $\mathrm{dc} / \mathrm{dc}$ converters. IEEE Trans. Circuits and Systems-I: Fundamental Theory and Applications, 45(2):133-141, February 1998.

[6] S. Y. R. Hui, Y. Shrivastava, S. Sathiakumar, K. K. Tse, and H. S.-H. Chung. A comparison of nondeterministic and deterministic switching methods of dc-dc power converter. IEEE Trans. Power Electronics, 13(6):1046-1055, November 1998.

[7] J. L. Rodríguez Marrero, J. M. Font, and G. C. Verghese. Analysis of the chaotic regime for $\mathrm{dc}-\mathrm{dc}$ converters under current-mode control. In Power Electronics Specialists' Conference, PESC 1996, pages 1477-1483, Baveno, Italy, June 1996.

[8] J. L. Rodríguez Marrero, R. Santos Bueno, and G. C. Verghese. Analysis and control of chaotic dc-dc switching power converters. In International Symposium on Circuits and Systems, ISCAS 1999, pages V-287-V-292, Orlando, Florida, USA, May/June 1999.

[9] A. M. Stanković, G. C. Verghese, and D. J. Perreault. Analysis and synthesis of randomized modulation schemes for power converter. IEEE Trans. Power Electronics, 10(6):680-693, November 1995 .

[10] G. Yuan, S. Banerjee, E. Ott, and J. A. Yorke. Border-collision bifurcations in the buck converter. IEEE Trans. Circuits and Systems-I: Fundamental Theory and Applications, 45(7):707-715, July 1998.

Oliver Woywode He was born in Schwerin, Germany in May 1968. He received the M.S. degree from
Portland State University, Oregon in Electrical and Computer Engineering in 1996 and the Dipl.-Ing. from TU Dresden in Elektrotechnik in 1997. He is a member of Eta Kappa $\mathrm{Nu}$ and a student member of IEEE. From 1992 till $1997 \mathrm{Mr}$. Woywode received a stipend from Friedrich-Naumann-Stiftung. He was a Fulbright grantee in the U.S. from fall 1994 till spring 1996. He is receiving a Sächsiches Landesstipendium and is currently working toward the Ph.D. degree in Elektrotechnik at TUD. His research interests include theory of nonlinear dynamics and applications in Power Electronics and Power Semiconductor devices.

Henry Güldner He was born in Demern, Germany in March 1941. He received the Dipl.-Ing., Dr.-Ing. (Ph.D.) and Dr.-Ing. habil. degrees all from TU Dresden, Germany in Electrical Engineering in 1967, 1971 and 1979 respectively. Univ.-Prof. Dr.-Ing. habil. Henry Güldner is a member of VDE and IEEE. He has been a Research Assistant (1967-1971) and an Assistant Professor (1971-1976) at TUD. From 1976-1982 he worked in the Institut für Mikroelektronik Dresden (IMD). From 1982-1989 he was an Associate Professor for Electrical Engineering at TUD. He was full Professor for Power Electronics at the Hochschule für Verkehrswesen Dresden from 1989-1993. Since 1993 he has been Univ.-Professor for Power Electronics at TUD. His main research interests are in power electronics, modelling of power electronic devices and systems, AC drives and electronic ballast. 